\title{
X-ray Absorption Spectroscopy of Transition Metal-Magnesium Hydride Thin Films
}

\author{
T. J. Richardson ${ }^{\mathrm{a}, *}$, B. Farangis ${ }^{\mathrm{a}}$, J. L. Slack ${ }^{\mathrm{a}}$, P. Nachimuthu ${ }^{\mathrm{b}}$, \\ R. Pereira ${ }^{\mathrm{b}}$, N. Tamura ${ }^{\mathrm{b}}$, and M. Rubin ${ }^{\mathrm{a}}$ \\ ${ }^{a}$ Environmental Energy Technologies Division, \\ ${ }^{\mathrm{b}}$ Advanced Light Source, \\ Ernest Orlando Lawrence Berkeley National Laboratory \\ Berkeley, California 94720, USA
}

*Corresponding author, E-mail address: tjrichardson@lbl.gov

\begin{abstract}
Mixed metal thin films containing magnesium and a first-row transition element exhibit very large changes in both reflectance and transmittance on exposure to hydrogen gas. Changes in electronic structure and coordination of the magnesium and transition metal atoms during hydrogen absorption were studied using dynamic in situ transmission mode X-ray absorption spectroscopy. Mg K-edge and $\mathrm{Ni}$, Co, and $\mathrm{Ti}$ L-edge spectra reflect both reversible and irreversible changes in the metal environments. A significant shift in the nickel L absorption edge shows it to be an active participant in hydride formation. The effect on cobalt and titanium is much less dramatic, suggesting that these metals act primarily as catalysts for formation of magnesium hydride.
\end{abstract}

Keywords: A. hydrogen storage materials, thin films; C. EXAFS, NEXAFS, X-ray diffraction. 


\section{Introduction}

Mixed metal thin films containing magnesium and a first-row transition element $[1,2]$ can exhibit very large changes in both reflectance and transmittance when hydrogen is absorbed or released. Switchable mirrors based on this phenomenon may be useful in a variety of consumer and industrial applications, including smart windows, electronic displays, and satellite heat control. A knowledge of the chemical and physical changes that accompany the optical transitions is essential for optimizing the characteristics of the mirror and transparent phases, the range of switching, and the durability of switchable mirror devices. While previous work using infrared spectroscopy has shown that ternary hydrides such as $\mathrm{Mg}_{2} \mathrm{NiH}_{4}$ are formed during hydriding of $\mathrm{Ni}-\mathrm{Mg}, \mathrm{Mn}-\mathrm{Mg}, \mathrm{Co}-\mathrm{Mg}$, and Fe-Mg films, the extent of transition metal consumption by these reactions is unclear. Binary transition metal hydrides or unreacted metals or alloys may also be present. In addition, similar switching behavior has been found in other systems (Ti-Mg, V-Mg, Nb-Mg and pure $\mathrm{Mg}$ films) in which ternary hydrides are not known to form. In situ X-ray absorption spectroscopy (XRAS) is well suited to investigation of hydride formation because it provides element-specific information about valence states and nearneighbor interactions, and because all phases containing that element contribute to the spectra.

\section{Experimental}

Mixed metal thin films containing Ni, Co, and Ti with Mg were deposited by DC magnetron co-sputtering from separate $5 \mathrm{~cm}$ diameter transition metal and $\mathrm{Mg}$ targets. The base pressure was $1.4 \times 10^{-7}$ Torr, process pressure $2 \mathrm{mTorr}(\mathrm{Ar})$, target-to-substrate distance $7.5 \mathrm{~cm}$. Power levels were adjusted to produce films with $\mathrm{Mg}$ :transition metal ratios ranging from 2 to 10, as measured by Rutherford backscattering spectrometry. A 5-10 nm Pd over-layer was 
applied to each sample at 10 mTorr Ar pressure, Pd power 12 watts. Film thicknesses were measured by stylus profilometry. Samples for transmission mode XRAS were deposited on 100 nm thick silicon nitride membranes (Structure Probe, Inc. West Chester, PA). XRAS experiments were performed on Beamline 6.3.1 at the Advanced Light Source (ALS). Samples for X-ray diffraction (XRD) were prepared on silicon wafers. XRD experiments were carried out using monochromatic $8.0478 \mathrm{eV}$ incident radiation on ALS Beamline 7.3.3.

\section{Results and discussion}

\subsection{XRD investigation}

Mixed metal films deposited on unheated substrates were amorphous by XRD. To improve the crystallinity, a freshly-deposited $1 \mu \mathrm{m}$ thick Mg-Ni film (Mg:Ni ca. 4) was annealed for $1 \mathrm{~h}$ at $125^{\circ} \mathrm{C}$ in vacuo prior to Pd deposition (to avoid alloying of $\mathrm{Pd}$ and $\mathrm{Mg}$ ). A mixture of $4 \%$ hydrogen in helium was directed across the film surface at ambient temperature using a laminar flow jig. XRD patterns were recorded at five-minute intervals during hydrogenation. The beginning and ending patterns are shown in the large frame in Figure 1. The unreacted film exhibited strong 003,006 , and $206 \mathrm{Mg}_{2} \mathrm{Ni}$ reflections [3] near $21^{\circ}, 41^{\circ}$, and $59^{\circ}$, respectively, due to strong preferred orientation of the intermetallic phase, along with broad reflections due to $\operatorname{Pd}$ at $40^{\circ}, 48^{\circ}$, and $70^{\circ}$. The precision of the peak positions is poor due to slight misalignment of the diffractometer. No reflections for Mg were observed. As the sample began to absorb hydrogen, the $\mathrm{Mg}_{2} \mathrm{Ni}$ peaks (exemplified by the 003 reflection, insert) first shifted to lower angle due to the slight c-axis expansion during formation of the interstitial hydride $\mathrm{Mg}_{2} \mathrm{NiH}_{0.3}$ [3], then gradually decreased in intensity. The Pd peaks also shifted to lower angles as $\mathrm{PdH}_{\mathrm{x}}$ was formed. No new reflections appeared. On exposure to air, the film returned to the mirror state, but 
remained amorphous. The XRD pattern did not change during subsequent hydrogen loading and unloading cycles.

\subsection{XRAS investigations}

\subsubsection{Mg-Ni films}

The development of the Ni L-edge spectrum (Fig. 2) during hydrogen loading of a Mg-Ni film (Mg:Ni ca. 6) showed similar features. There was initially a small shift in the $\mathrm{L}_{3}$ and $\mathrm{L}_{2}$ peak positions, followed by a simultaneous decrease in intensity at this position and increase in intensity at a new position. The edge shift at the end of hydrogenation was about $2.5 \mathrm{eV}$, consistent with conversion from a metallic to a semiconducting state (e.g. $\mathrm{Mg}_{2} \mathrm{NiH}_{4}$ ), but somewhat larger than that predicted by ab initio calculations [4]. Exposure of the hydrided film to air for $24 \mathrm{~h}$ caused the spectrum to return to the first intermediate state, which is presumably the very stable $\mathrm{Mg}_{2} \mathrm{NiH}_{0.3}$.

The Mg K-edge spectra for an identical film are shown in Figure 3. An edge shift similar to that for Ni occurred on hydrogen loading, followed by a partial return to the original state after air exposure. Magnesium was present in the fresh film as both $\mathrm{Mg}_{2} \mathrm{Ni}$ and $\mathrm{Mg}$. The complete conversion to hydrides is shown by the absence of a shoulder on the low-energy side of the absorption edge. The presence of this edge in the air-exposed spectrum shows that some $\mathrm{Mg}$ has returned to the metallic state, while some may still be present as $\mathrm{MgH}_{2}$ and $\mathrm{Mg}_{2} \mathrm{NiH}_{0.3}$.

\subsubsection{Mg-Co films}

Cobalt does not alloy with magnesium, but forms semiconducting $\mathrm{Mg}_{2} \mathrm{CoH}_{5}$ [5] and metallic $\mathrm{Mg}_{6} \mathrm{Co}_{2} \mathrm{H}_{11}$ [6]. Although the hydrogen-induced changes in optical properties of $\mathrm{Mg}-\mathrm{Co}$ 
films (Mg:Co ca. 6) were quite similar to those of Mg-Ni films, the changes in their in situ Co Ledge spectra (Fig. 4) were simpler and less dramatic. The shift was gradual, reaching a maximum of about $1 \mathrm{eV}$, a smaller shift than expected for formation of [7]. This suggests that most of the changes in the visible and near infrared were due to cobalt-catalyzed conversion of $\mathrm{Mg}$ to $\mathrm{MgH}_{2}$, and that either Co was not converted to $\mathrm{Mg}_{2} \mathrm{CoH}_{5}$ under these conditions, or that the Co L-edge shift does not fully reflect the difference in electronic structure between the metal and the ternary hydride. Features in the Mg K EXAFS region for both Mg-Ni and Mg-Co films were poorly resolved due to the presence of two or more Mg-containing phases.

\subsubsection{Mg-Ti films}

Titanium forms neither an alloy nor a ternary hydride with magnesium. There is a slight negative energy shift in the Ti L-edge spectrum (Fig. 5) of a pure, (Pd-coated) Ti thin film and that of a mixed Mg-Ti film. On absorbing hydrogen, the film becomes transparent, but only a small positive shift in the Ti L-edge spectrum occurs, despite the fact that in $4 \% \mathrm{H}_{2}$ at ambient temperature, complete conversion to $\mathrm{TiH}_{2}$ is expected [8]. Attempts to obtain a reference spectrum of $\mathrm{TiH}_{2}$ failed due to extensive surface oxidation (not seen in our films). Because all of the magnesium is present as either $\mathrm{Mg}$ or $\mathrm{MgH}_{2}$, the $\mathrm{Mg} \mathrm{K}$-edge spectra (Fig. 6) clearly show the effect of hydrogen loading on the absorption edge and the EXAFS oscillations, which are visible in the hydride state, but significantly damped both by the presence of weakly-scattering hydrogen atoms as Mg nearest neighbors and by disorder induced by the phase transformation and volume expansion. 


\section{Conclusions}

In situ XRD and XRAS spectra of mixed metal films are an effective tool for studying the structural and electronic transitions that occur during hydrogen loading. A sampling of results are presented here. The participation of magnesium in formation of binary and ternary hydrides in $\mathrm{Mg}-\mathrm{Ni}$ films contrasts with its (Ti-catalyzed) reaction to form pure $\mathrm{MgH}_{2}$ in $\mathrm{Mg}-\mathrm{Ti}$ films. Even when crystalline $\mathrm{Mg}_{2} \mathrm{Ni}$ is present before hydriding, the conversion of this phase to $\mathrm{Mg}_{2} \mathrm{NiH}_{4}$ renders the film amorphous by $\mathrm{XRD}$, and the crystallinity is not recovered on return to the mirror state. The interstitial hydride $\mathrm{Mg}_{2} \mathrm{NiH}_{0.3}$ is evident in both XRD and XRAS experiments at the beginning of hydriding, and in the air-exposed film by XRAS. The state of Co in Mg-Co films is somewhat less clear, and requires further study. In Mg-Ti films, optical switching does not appear to require complete conversion of $\mathrm{Ti}$ to $\mathrm{TiH}_{2}$, though this is not ruled out. In-depth reports on each of these systems will be the subject of future publications.

\section{Acknowledgement}

This work was supported by the Assistant Secretary for Energy Efficiency and Renewable Energy, Office of Building Technology, State and Community Programs, Office of Building Research and Standards of the U.S. Department of Energy under Contract No. DE-AC0376SF00098. 


\section{References}

[1] T. J. Richardson, J. L. Slack, R. D. Armitage, R. Kostecki, B. Farangis, M. D. Rubin, Appl. Phys. Lett. 78 (2001) 3047.

[2] T. J. Richardson, J. L. Slack, B. Farangis, M. D. Rubin, Appl. Phys. Lett. 80 (2002) 1349.

[3] J. Schefer, P. Fischer, W. Hälg, F. Stucki, L. Schlapbach, J. J. Didisheim, K. Yvon, A. F. Andresen, J. Less-Common Metals 74 (1980) 65.

[4] W. R. Myers, L-W. Wang, T. J. Richardson, M. D. Rubin, J. Appl. Phys. 91 (2002) 4879.

[5] P. Zolliker, K. Yvon, P. Fischer, and J. Schefer, Inorg. Chem. 24, (1985) 4177.

[6] R. Cerny, F. Bonhomme, K. Yvon, P. Fischer, P. Zolliker, D. E. Cox, and A Hewat, J. Alloys Compd. 187, (1992) 233.

[7] E. Belin, M. Gupta, P. Zolliker, and K. Yvon, J. Less-Common Met. 130, (1987) 267.

[8] E. Nowicka, Vacuum 48 (1997) 199. 


\section{Figure captions}

1. X-ray diffraction pattern of $1 \mu \mathrm{m} \mathrm{Mg-Ni}$ film on Si during hydrogen absorption.

2. Ni L-edge X-ray absorption spectra of Mg-Ni film during hydrogen absorption.

3. Mg K-edge X-ray absorption spectra of Mg-Ni film before and after hydrogen absorption, and after exposure to air.

4. Co L-edge X-ray absorption spectra of $\mathrm{Mg}$-Co before and after hydrogen absorption.

5. Ti L-edge X-ray absorption spectra of a pure Ti film and a Mg-Ti film before and after hydrogen absorption.

6. Mg K-edge EXAFS spectra of Mg-Ti film before and after hydrogen absorption. 


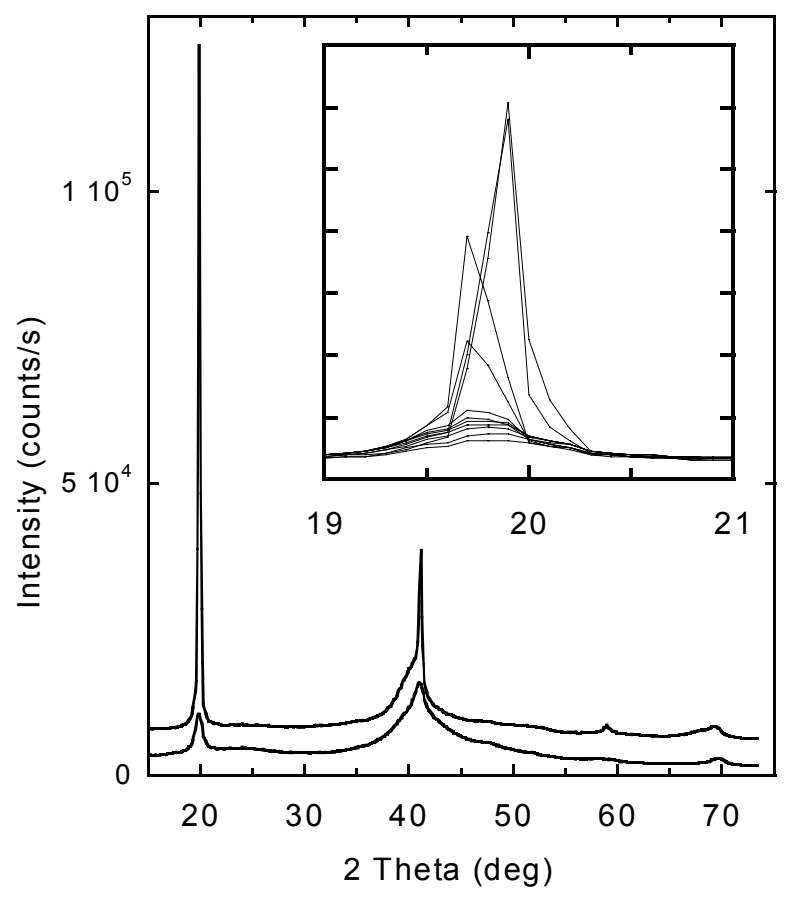

Richardson et al. Figure 1 


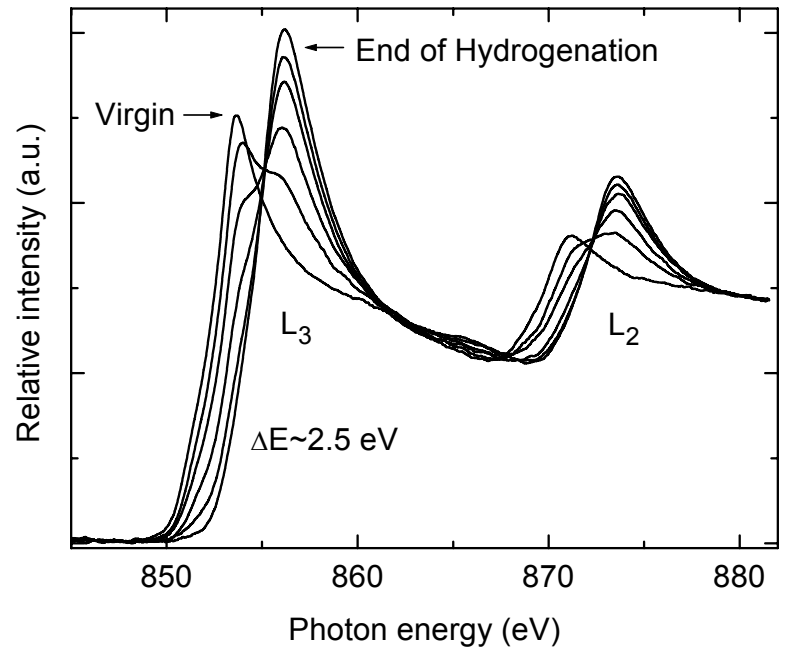

Richardson et al. Figure 2 


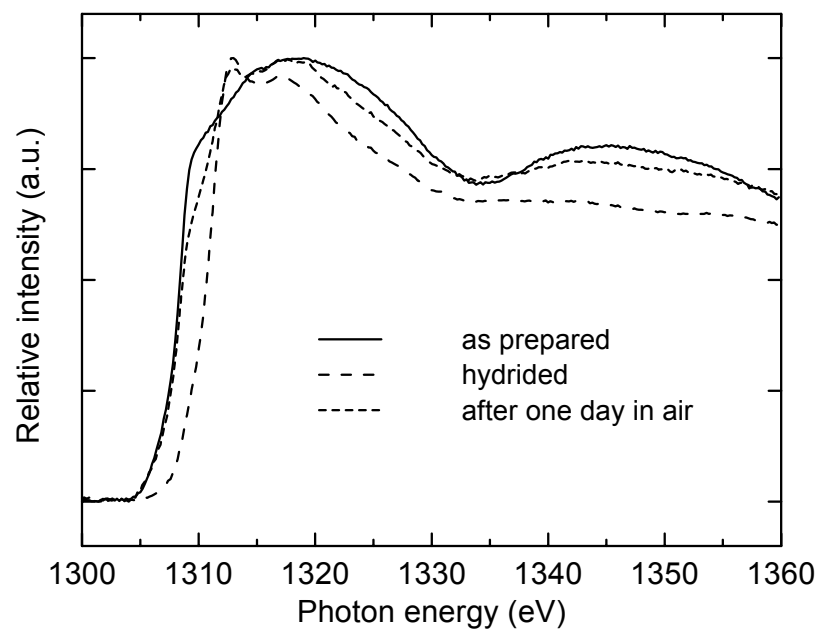

Richardson et al. Figure 3 


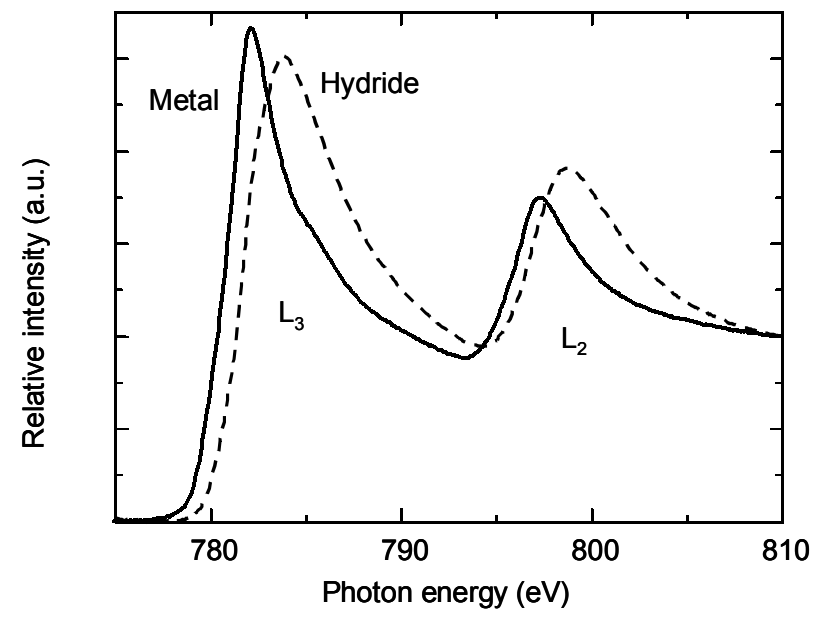

Richardson et al. Figure 4 


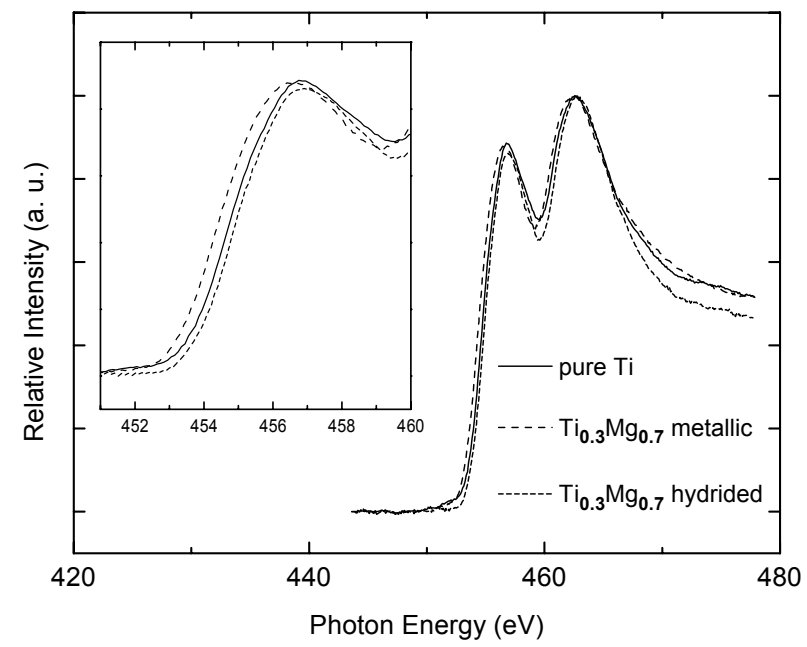

Richardson et al. Figure 5 


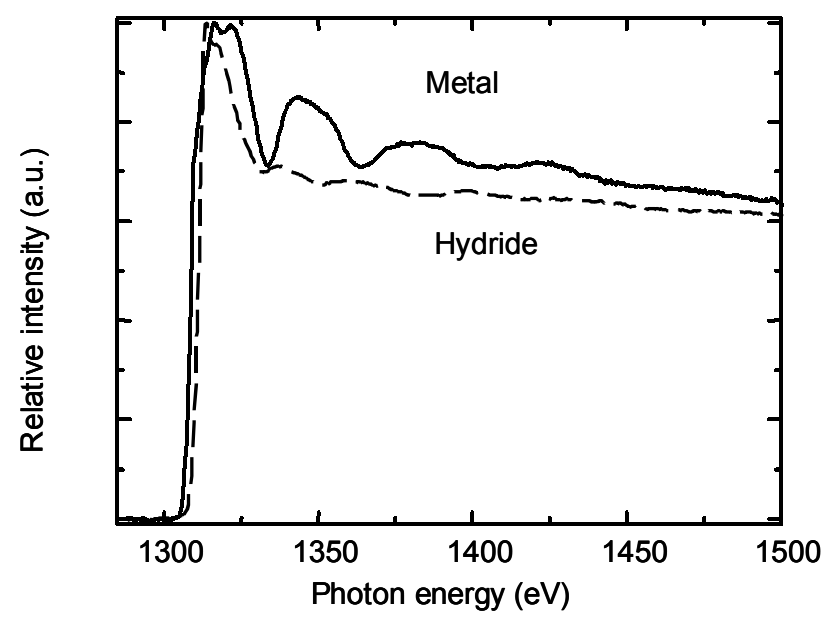

Richardson et al. Figure 6 